PROCEEDINGS OF THE

AMERICAN MATHEMATICAL SOCIETY

Volume 131, Number 11, Pages 3477-3485

S 0002-9939(03)06908-9

Article electronically published on February 6, 2003

\title{
THE RANK OF FINITELY GENERATED MODULES OVER GROUP ALGEBRAS
}

\author{
GÁBOR ELEK \\ (Communicated by Martin Lorenz)
}

\begin{abstract}
We show the existence of a rank function on finitely generated modules over group algebras $K \Gamma$, where $K$ is an arbitrary field and $\Gamma$ is a finitely generated amenable group. This extends a result of W. Lück (1998).
\end{abstract}

\section{INTRODUCTION}

The goal of this paper is to construct a real-valued non-trivial rank function on finitely generated modules over a group algebra $K \Gamma$, where $K$ is an arbitrary field and $\Gamma$ is a finitely generated amenable group.

Theorem 1. Let $K$ be a field and let $\Gamma$ be a finitely generated amenable group. Then there exists a function

$$
\text { rk }: \text { Fin.Gen.Modules }(K \Gamma) \rightarrow \mathbb{R}
$$

such that

(1) If $M \simeq N$, then $r k(N)=\operatorname{rk}(M)$.

(2) If $0 \rightarrow M \rightarrow P \rightarrow N \rightarrow 0$ is an exact sequence, then $r k(M)+r k(N)=$ $r k(P)$.

(3) $\operatorname{rk}(K \Gamma)=1$.

The existence of such rank function was proved by Lück [2] in the case when $K$ is the field of complex numbers. In 2, for any finitely generated module $M$ over $\mathbb{C} \Gamma$ the author associated an invariant subspace $V_{M}$ of the Hilbert space $\left[l^{2}(\Gamma)\right]^{n}$. The rank of the module $M$ was defined as the von Neumann dimension of $V_{M}$. In an earlier paper [1] we extended Lück's result for finite fields using the notion of topological entropy. In the general case, we will apply the following strategy. For any finitely generated module $M$ over $K \Gamma$, we associate an invariant subspace $V_{M}$ of the function field $\prod_{\gamma \in \Gamma} K^{n}$. Then we define an average dimension for such invariant spaces that behaves similarly to the von Neumann dimension. The rank of $M$ will be the average dimension of the associated invariant subspace $V_{M}$. An observation of Lück suggests that Theorem 1 might serve as a ring theoretic characterisation of amenability. That is, such a rank function may not exist if $\Gamma$ is a non-amenable group.

Received by the editors November 14, 2001 and, in revised form, May 31, 2002

2000 Mathematics Subject Classification. Primary 43A07, $20 \mathrm{C} 07$.

Key words and phrases. Amenable groups, group algebras, finitely generated modules, invariant subspaces. 


\section{INVARIANT SUBSPACES}

Let $K$ be an arbitrary field equipped with the discrete topology and let $\Gamma$ be a countable group. For any integer $n \geq 1$, one can consider $\prod_{\gamma \in \Gamma} K^{n}$, the space of vector-valued functions with the product topology. The space $\prod_{\gamma \in \Gamma} K^{n}$ is metrizable and $\left\{f_{m}\right\}_{m=1}^{\infty} \rightarrow f$ if for any $\gamma \in \Gamma, f_{m}(\gamma)=f(\gamma)$ for sufficiently large $m$ depending on $\gamma$. The natural right $\Gamma$-action on $\prod_{\gamma \in \Gamma} K^{n}$ is defined by

$$
(f \cdot \gamma)(\delta)=f\left(\delta \gamma^{-1}\right) \text {. }
$$

Note that we also have a left $\Gamma$-action on $\prod_{\gamma \in \Gamma} K^{n}$ commuting with the right $\Gamma$-action:

$$
(\gamma \cdot f)(\delta)=f\left(\gamma^{-1} \delta\right)
$$

Both the left and right $\Gamma$-actions are continuous and $K$-linear. Denote by $K \Gamma$ the group algebra of $\Gamma$ over $K$. The left $\Gamma$-action defines a representation of $K \Gamma$ in the homomorphism ring of $\prod_{\gamma \in \Gamma} K^{n}$. A subspace $V \subseteq \prod_{\gamma \in \Gamma} K^{n}$ is called invariant if it is closed, $K$-linear and invariant under the right $\Gamma$-action.

\section{3. $\Gamma$-EQUIVARIANT MAPS}

Let us consider $M a t_{n \times m}(K \Gamma)$ the space of finitely supported $M a t_{n \times m}(K)$-valued functions on $\Gamma$. If $M=\sum_{\gamma \in \Gamma} M(\gamma) \gamma \in M a t_{n \times m}(K \Gamma)$ and $f \in \prod_{\gamma \in \Gamma} K^{m}$, then the matrix multiplication $M: \prod_{\gamma \in \Gamma} K^{m} \rightarrow \prod_{\gamma \in \Gamma} K^{n}$ is defined by

$$
M f(\delta)=\sum_{\gamma \in \Gamma} M(\gamma) f\left(\gamma^{-1} \delta\right) \in K^{n} .
$$

Note that $M: \prod_{\gamma \in \Gamma} K^{m} \rightarrow \prod_{\gamma \in \Gamma} K^{n}$ is continuous, $K$-linear and $\Gamma$-equivariant, that is, it commutes with the right $\Gamma$-action.

Proposition 3.1. Let $V \subseteq \prod_{\gamma \in \Gamma} K^{m}, W \subseteq \prod_{\gamma \in \Gamma} K^{n}$ be invariant subspaces and let $T: V \rightarrow W$ be a continuous $K$-linear $\Gamma$-equivariant map. Then there exists $M_{T} \in M_{a x} t_{n \times m}(K \Gamma)$ such that for any $v \in V, M_{T} v=T v$.

Proof. Let $e_{\Gamma}$ be the unit element of $\Gamma$. Note that if $T_{1}$ and $T_{2}$ are $\Gamma$-equivariant maps and $T_{1} v\left(e_{\Gamma}\right)$ and $T_{2} v\left(e_{\Gamma}\right)$ for all $v \in V$, then $T_{1} v=T_{2} v$ for all $v \in V$. Since $T$ is continuous, there exists a finite subset $A \subseteq \Gamma$ such that $T v\left(e_{\Gamma}\right)$ depends only on the values of $v$ at the elements of $A$. Since

$$
\operatorname{Hom}_{K}\left(\bigoplus_{\gamma \in A} K^{m}, K^{n}\right)=\bigoplus_{\gamma \in A} \operatorname{Hom}_{K}\left(K^{m}, K^{n}\right),
$$

it is easy to see that there exist elements $\left\{M_{\gamma}\right\}_{\gamma \in A} \subseteq M_{a t_{n \times m}}(K)$ such that $T v\left(e_{\Gamma}\right)=\sum_{\gamma \in A} M_{\gamma} \cdot v(\gamma)$, for any $v \in V$. Let $M_{T}(\gamma)=M_{\gamma^{-1}}$. Then $T v\left(e_{\Gamma}\right)=$ $M_{T} v\left(e_{\Gamma}\right)$, for any $v \in V$.

\section{Duality}

In this section we study the baby-version of Pontrjagin's duality. All proofs are straightforward and are left to the reader. Let $\sum_{\gamma \in \Gamma} K^{n}$ be the space of finitely supported vector-valued functions on $\Gamma$ equipped with the discrete topology. Then one has the pairing

$$
\langle,\rangle: \prod_{\gamma \in \Gamma} K^{n} \times \sum_{\gamma \in \Gamma} K^{n} \rightarrow K
$$


defined by $\langle v, f\rangle=\sum_{\gamma \in \Gamma}(v(\gamma), f(\gamma))$, where (,) is the usual scalar product on $K^{n}$. Denote by $\left(\sum_{\gamma \in \Gamma} K^{n}\right)^{*}$ the space of all $K$-linear maps $T: \sum_{\gamma \in \Gamma} K^{n} \rightarrow K$. If $U \subseteq \sum_{\gamma \in \Gamma} K^{n}$ is a $K$-linear subspace, then its orthogonal set $U^{\perp}$ is defined as

$$
U^{\perp}=\left\{v \in \prod_{\gamma \in \Gamma} K^{n}:\langle v, f\rangle=0, \text { for any } f \in U\right\} .
$$

Note that $U^{\perp}$ is a closed $K$-linear subspace in $\prod_{\gamma \in \Gamma} K^{n}$. Similarly, if $W \subseteq$ $\prod_{\gamma \in \Gamma} K^{n}$, then

$$
W^{\perp}=\left\{f \in \sum_{\gamma \in \Gamma} K^{n}:\langle v, f\rangle=0, \text { for any } v \in W\right\} .
$$

Lemma 4.1. Let $W \subseteq \prod_{\gamma \in \Gamma} K^{n}$ be a $K$-linear subspace. Then $\left(W^{\perp}\right)^{\perp}=\bar{W}$, the closure of $W$ in $\prod_{\gamma \in \Gamma} K^{n}$.

Proof. It is enough to prove that if $w \notin \bar{W}$, then there exists $f \in W^{\perp}$ such that $\langle w, f\rangle \neq 0$. Since $w \notin \bar{W}$, there exists a finite subset $A \subseteq \Gamma$ such that $\left.\left.w\right|_{A} \notin W\right|_{A}$. Therefore there exists an element $f \in \sum_{\gamma \in \Gamma} K^{n}$ supported on $A$ that is orthogonal to $W$ and has a non-zero scalar product with $w$.

The weak topology of $\left(\sum_{\gamma \in \Gamma} K^{n}\right)^{*}$ can be defined the usual way via base sets. Let $T \in\left(\sum_{\gamma \in \Gamma} K^{n}\right)^{*}$ and let $f_{1}, f_{2}, \ldots, f_{r} \in \sum_{\gamma \in \Gamma} K^{n}$. Then,

$$
N_{T, f_{1}, f_{2}, \ldots, f_{r}}=\left\{S \in\left(\sum_{\gamma \in \Gamma} K^{n}\right)^{*}: T f_{i}=S f_{i}, \text { for all } i, 1 \leq i \leq r\right\} .
$$

Proposition 4.1. There exists a continuous isomorphism $\Psi$ between $\prod_{\gamma \in \Gamma} K^{n}$ and $\left(\sum_{\gamma \in \Gamma} K^{n}\right)^{*}$, defined by $\Psi(v)=\langle v,$.$\rangle . Similarly, if A \subseteq \sum_{\gamma \in \Gamma} K^{n}$ is a K-linear subspace, then $\Psi_{A}(v)=\langle v,$.$\rangle defines a continuous isomorphism between A^{\perp}$ and $\left(\sum_{\gamma \in \Gamma} K^{n} / A\right)^{*}$.

Let $A, B \subseteq \sum_{\gamma \in \Gamma} K^{n}$ be $K$-linear subspaces and $T: \sum_{\gamma \in \Gamma} K^{n} / A \rightarrow \sum_{\gamma \in \Gamma} K^{n} / B$ a $K$-linear transformation. Then the adjoint of $T$ is the well-defined continuous map $T^{*}: B^{\perp} \rightarrow A^{\perp}$ such that $\left\langle T^{*} v, f\right\rangle=\langle v, T f\rangle$, for any $v \in B^{\perp}$ and $f \in \sum_{\gamma \in \Gamma} K^{n} / A$. Let us suppose that $A \subseteq \sum_{\gamma \in \Gamma} K^{n}$ is invariant under the right $\Gamma$-action. Then $A^{\perp}$ is an invariant subspace. Also, if $T$ is $\Gamma$-equivariant, then $T^{*}$ is $\Gamma$-equivariant as well. Indeed, the adjoint of the right translation by $\gamma \in \Gamma$ is just the right translation by $\gamma^{-1}$. Observe that $\sum_{\gamma \in \Gamma} K^{n}$ can be identified with $(K \Gamma)^{n}$ as a right $K \Gamma$-module. The subspaces $A \in \sum_{\gamma \in \Gamma} K^{n}$ which are invariant under the right $\Gamma$-action can be identified with the $K \Gamma$-submodules. Hence the spaces $\sum_{\gamma \in \Gamma} K^{n} / A$ can be identified with finitely generated right $K \Gamma$-modules. We can formulate the previous observations in the following proposition.

Proposition 4.2. The duality functor $M \rightarrow M^{*}$ associates an invariant subspace to a finitely generated $K \Gamma$-module. The adjoint functor $T \rightarrow T^{*}$ associates a $\Gamma$ equivariant continuous map between invariant subspaces to any module homomorphism.

Later we will see that if $M$ and $N$ are isomorphic modules, then $M^{*}$ and $N^{*}$ are isomorpic as well; also if $T$ is an isomorphism, then $T^{*}$ is an isomorphism as well. 


\section{WEAK EXACTNESS}

Proposition 5.1. Let $0 \rightarrow M \stackrel{i}{\rightarrow} P \stackrel{\pi}{\rightarrow} N \rightarrow 0$ be an exact sequence of finitely generated right $K \Gamma$-modules. Then $0 \rightarrow N^{*} \stackrel{\pi^{*}}{\rightarrow} P^{*} \stackrel{i^{*}}{\rightarrow} M^{*} \rightarrow 0$ is weak exact, that is,

(1) $\pi^{*}$ is injective.

(2) $M^{*}=\overline{\operatorname{Ran}^{*}}$

(3) $\operatorname{Ker}^{*}=\overline{\operatorname{Ran} \pi^{*}}$.

Proof. (1) If $\pi^{*}(f)=0$, then $\left\langle\pi^{*}(f), p\right\rangle=0$ for any $p \in P$. That is, $\langle f, \pi(p)\rangle=0$. Hence $\langle f, n\rangle=0$ for any $n \in N$. Therefore $f=0$. (2) Let $\left\langle i^{*}(g), m\right\rangle=0$, for any $g \in P^{*}$. Then $\langle g, i(m)\rangle=0$, thus $m$ must be 0 . Therefore $i^{*}(g)$ is dense in $M^{*}$, by Lemma 4.1. (3) $\left\langle i^{*} \circ \pi^{*}(f), m\right\rangle=\langle f, \pi \circ i(m)\rangle=0$. This shows that $\operatorname{Ran} \pi^{*} \subseteq \operatorname{Ker} i^{*}$. Since $\operatorname{Ker} i^{*}$ is closed, it means that $\overline{\operatorname{Ran} \pi^{*}} \subseteq \operatorname{Ker} i^{*}$. Now suppose that $p \perp \operatorname{Ran} \pi^{*}$. By Lemma 4.1, it is enough to see that $p \perp \operatorname{Ker} i^{*}$ as well. However, $0=\left\langle p, \pi^{*}(f)\right\rangle=\langle\pi(p), f\rangle$, that is, $\pi(p)=0$. Hence by the exactness, $p=i(m)$. Now $\langle i(m), g\rangle=0$ if $g \in \operatorname{Ker} i^{*}$.

\section{Amenability}

Let us recall the important notion of quasi-tilings of amenable groups following the paper of Ornstein \& Weiss [3]. Let $\Gamma$ be a finitely generated group with symmetric generating set $\left\{g_{1}, g_{2}, \ldots, g_{k}\right\}$. The generators determine a word-metric $d$ on $\Gamma$ in such a way that the right multiplications by the elements of $\Gamma$ are isometries. The group $\Gamma$ is called amenable if it has an exhaustion

$$
\mathcal{F}_{1} \subseteq \mathcal{F}_{2} \subseteq \cdots \bigcup_{k=1}^{\infty} \mathcal{F}_{k}=\Gamma
$$

by finite subsets such that for any fixed $r \in \mathbb{N}$,

$$
\lim _{k \rightarrow \infty} \frac{\left|\partial_{r} \mathcal{F}_{k}\right|}{\left|\mathcal{F}_{k}\right|}=0
$$

where $\partial_{r} \mathcal{F}_{k}=\left\{\gamma \in \Gamma: d\left(\gamma, \mathcal{F}_{k}\right) \leq r\right.$ and $\left.d\left(\gamma, \Gamma-\mathcal{F}_{k}\right) \leq r\right\}$. Such exhaustions are called Følner-exhaustions. The finite subsets $A_{1}, A_{2}, \ldots, A_{k} \subseteq \Gamma$ are called $\epsilon$-disjoint if there are subsets $B_{1}, B_{2}, \ldots, B_{k} \subseteq \Gamma$ such that

(1) $B_{i} \subseteq A_{i}$ for any $i=1,2, \ldots, k$.

(2) $\frac{\left|B_{i}\right|}{\left|A_{i}\right|}>1-\epsilon$

(3) $\left|B_{i} \cap B_{j}\right|=\emptyset$ if $i \neq j$.

Also, we say that the finite subsets $A_{1}, A_{2}, \ldots, A_{k} \subseteq \Gamma \delta$-covers the set $A \subseteq \Gamma$, if

$$
\frac{\left|A \cap\left(\bigcup_{i=1}^{k} A_{i}\right)\right|}{|A|} \geq \delta .
$$

Finally, the finite subsets $A_{1}, A_{2}, \ldots, A_{k} \subseteq \Gamma \epsilon$-quasitile the set $A \subseteq \Gamma$, if there are finite sets $C_{1}, C_{2}, \ldots, C_{k} \subseteq \Gamma$ such that

(1) $A_{i} C_{i} \subseteq A$, for any $i=1,2, \ldots, k$.

(2) $A_{i} C_{i} \cap A_{j} C_{j}=\emptyset$ if $i \neq j$.

(3) $\left\{A_{i} c: c \in C_{i}\right\}$ form an $\epsilon$-disjoint family.

(4) $\left\{A_{i} C_{i}, i=1,2, \ldots, k\right\}$ form a $(1-\epsilon)$-cover of $A$. 
The sets $C_{i}$ are called the tiling centers. The following result is due to Ornstein \& Weiss [3]:

Proposition 6.1. Let $\mathcal{F}_{1} \subseteq \mathcal{F}_{2} \subseteq \ldots$ and $\mathcal{G}_{1} \subseteq \mathcal{G}_{2} \subseteq \ldots$ be Følner-exhaustions. Let $\epsilon \in\left(0, \frac{1}{4}\right)$ and $N>0$. Then there exist integers $N \leq n_{1}<n_{2}<\cdots<n_{l}$ and $M>0$ such that $\left\{\mathcal{F}_{n_{1}}, \mathcal{F}_{n_{2}}, \ldots, \mathcal{F}_{n_{l}}\right\}$ e-quasitile $\mathcal{G}_{m}$ for any $m \geq M$.

\section{Average Dimension}

Let $B \subseteq \Gamma$ be a finite subset and let $V \subseteq \prod_{\gamma \in \Gamma} K^{n}$ be an invariant subset. Denote by $V_{B}$ the space of vectors $v$ supported on $B$ such that there exists $w \in V$, $\left.v\right|_{B}=\left.w\right|_{B}$. The average dimension, $\operatorname{dim}_{\Gamma}^{A}(V)$, is defined as

$$
\operatorname{dim}_{\Gamma}^{A}(V)=\limsup _{k \rightarrow \infty} \frac{\operatorname{dim} V_{\mathcal{F}_{k}}}{\left|\mathcal{F}_{k}\right|}
$$

We have the following proposition.

Proposition 7.1. The average dimension of an invariant subspace does not depend on the particular choice of the Følner-exhaustion and in fact,

$$
\operatorname{dim}_{\Gamma}^{A}(V)=\lim _{k \rightarrow \infty} \frac{\operatorname{dim} V_{\mathcal{F}_{k}}}{\left|\mathcal{F}_{k}\right|} .
$$

Proof. Let $\mathcal{F}_{1} \subseteq \mathcal{F}_{2} \subseteq \ldots$ and $\mathcal{G}_{1} \subseteq \mathcal{G}_{2} \subseteq \ldots$ be Følner-exhaustions. It is enough to prove that

$$
\limsup _{k \rightarrow \infty} \frac{\operatorname{dim} V_{\mathcal{F}_{k}}}{\left|\mathcal{F}_{k}\right|} \geq \limsup _{k \rightarrow \infty} \frac{\operatorname{dim} V_{\mathcal{G}_{k}}}{\left|\mathcal{G}_{k}\right|}
$$

First of all note that by sorting out a suitable subsequence we may suppose that

$$
\operatorname{dim}_{\Gamma}^{A}(V)=\lim _{k \rightarrow \infty} \frac{\operatorname{dim} V_{\mathcal{F}_{k}}}{\left|\mathcal{F}_{k}\right|}
$$

It is enough to see that for any $\delta>0$, there exists $Q>0$ such that if $q>Q$, then

$$
\frac{\operatorname{dim} V_{\mathcal{G}_{q}}}{\left|\mathcal{G}_{q}\right|} \leq(1+\delta) \operatorname{dim}_{\Gamma}^{A}(V)+\delta
$$

Pick an integer $N$ such that if $k>N$, then

$$
\frac{\operatorname{dim} V_{\mathcal{F}_{k}}}{\left|\mathcal{F}_{k}\right|} \leq \operatorname{dim}_{\Gamma}^{A}(V)+\epsilon,
$$

where the exact value of $\epsilon$ will be determined at the end of the proof. By Proposition 6.1, we have integers $N \leq n_{1}<n_{2} \cdots<n_{l}$ and $M>0$ such that $\left\{\mathcal{F}_{n_{1}}, \mathcal{F}_{n_{2}}, \ldots, \mathcal{F}_{n_{l}}\right\}$ $\epsilon$-quasitile $\mathcal{G}_{m}$ for any $m \geq M$. Denote by $S_{1}, S_{2}, \ldots, S_{c_{m}}$ the translated copies of Følner-sets used in the $\epsilon$-quasitiling of $\mathcal{G}_{m}$ and denote by $T_{1}, T_{2}, \ldots, T_{c_{m}}$ the $\epsilon$ disjoint parts of the $S_{i}$ 's as in the definition of $\epsilon$-disjoint sets. Then by the invariance of $V$, we have the following estimate for any $1 \leq i \leq c_{m}$ :

$$
\operatorname{dim} V_{T_{i}} \leq \operatorname{dim} V_{S_{i}} \leq\left|S_{i}\right|\left(\operatorname{dim}_{\Gamma}^{A}(V)+\epsilon\right) .
$$

Hence, $\operatorname{dim} V_{T_{i}} \leq(1-\epsilon)^{-1}\left|T_{i}\right|\left(\operatorname{dim}_{\Gamma}^{A}(V)+\epsilon\right)$. Also, $\left|\mathcal{G}_{m}\right| \geq \sum_{i=1}^{c_{m}}\left|T_{i}\right|$. By a straightforward pigeon-hole argument,

$$
\operatorname{dim} V_{\mathcal{G}_{m}} \leq \sum_{i=1}^{c_{m}} \operatorname{dim} V_{T_{i}}+\epsilon^{2} n\left|\mathcal{G}_{m}\right|
$$


Therefore,

$$
\operatorname{dim} V_{\mathcal{G}_{m}} \leq(1-\epsilon)^{-1}\left(\operatorname{dim}_{\Gamma}^{A}(V)+\epsilon\right)\left|\mathcal{G}_{m}\right|+\epsilon^{2} n\left|\mathcal{G}_{m}\right|,
$$

that is,

$$
\frac{\operatorname{dim} V_{\mathcal{G}_{m}}}{\left|\mathcal{G}_{m}\right|} \leq(1-\epsilon)^{-1}\left(\operatorname{dim}_{\Gamma}^{A}(V)+\epsilon\right)+\epsilon^{2} n .
$$

Choosing $\epsilon$ carefully, we can assert that (1) holds.

\section{EXTENDED CONFIGURATIONS}

In this section we use a technique that proved to be quite useful in the case of calculating topological entropies [4], [1. Let $V \subseteq \prod_{\gamma \in \Gamma} K^{n}$ be an invariant subspace. An extended configuration $V^{E}$ is defined the following way. For any finite set $B \subseteq \Gamma$, let $V_{B}^{E} \subseteq \prod_{\gamma \in \Gamma} K^{n}$ be a finite-dimensional $K$-linear vectorspace supported on $B$ that satisfies the following four conditions:

(1) $V_{B} \subseteq V_{B}^{E}$.

(2) $V_{B \gamma}^{E}=V_{B}^{E} \cdot \gamma$, for any $\gamma \in \Gamma$.

(3) If $B \subseteq C$ and $f \in V_{C}^{E}$, then there is a $g \in V_{B}^{E}$ such that $\left.f\right|_{B}=\left.g\right|_{B}$.

(4) If $v \in \prod_{\gamma \in \Gamma} K^{n}$ and for any finite set $B \subseteq \Gamma$ there exists $f \in V_{B}^{E}$ such that $\left.f\right|_{B}=\left.v\right|_{B}$, then $f \in V$.

We call such a system of functions an extended configuration of $V$. The average dimension of $V^{E}$ is defined as

$$
\operatorname{dim}_{\Gamma}^{A}\left(V^{E}\right)=\limsup _{k \rightarrow \infty} \frac{\operatorname{dim} V_{\mathcal{F}_{k}}^{E}}{\left|\mathcal{F}_{k}\right|} .
$$

For any $r>0$ and a finite set $A \subseteq \Gamma$ let

$$
B_{r}(A)=\{\gamma \in \Gamma: d(\gamma, A) \leq r\} .
$$

The following proposition is a vectorspace version of the well-known König lemma.

Proposition 8.1. Let $V^{E}$ be an extended configuration of an invariant vectorspace $V \subseteq \prod_{\gamma \in \Gamma} K^{n}$ and let $A \subseteq \Gamma$ be a finite set. Denote by $V_{A}^{r}$ the space of $K^{n}$-valued functions $f$ supported on $A$ such that there exists $v \in V_{B_{r}(A)}^{E}$ so that $\left.v\right|_{A}=\left.f\right|_{A}$. Then if $r$ is sufficiently large, depending on $A$, then $V_{A}^{r}=V_{A}$.

Proof. Let $C \subseteq \Gamma$ be a finite subset. Call a vector $v \in V_{C}^{E}$ extendable if for any finite set $C \subseteq D \subseteq \Gamma$ there exists $w \in V_{D}^{E}$ such that $\left.w\right|_{C}=\left.v\right|_{C}$. Denote the space of extendable vectors by $V_{C}^{\infty}$. Obviously, $V_{C} \subseteq V_{C}^{\infty}$. For $v \in V_{C}^{E}$, the height of $v$, $h(v)$ is defined as follows:

$$
h(v)=\sup _{r \geq 0}\left\{r: \text { there exists } w \in V_{B_{r}(C)}^{E} \text { such that }\left.w\right|_{C}=\left.v\right|_{C}\right\} .
$$

Note that $h(v)=\infty$ means that $v$ is extendable.

Lemma 8.1. If $v \in V_{C}^{\infty}$, then there exists $w \in V_{B_{1}(C)}^{\infty}$ such that $\left.v\right|_{C}=\left.w\right|_{C}$.

Proof. Denote by $H_{v}$ the affine space of vectors in $V_{B_{1}(C)}^{E}$ such that if $w \in H_{v}$, then $\left.w\right|_{C}=\left.v\right|_{C}$. Since $v$ is extendable, the height function is unbounded on $H_{v}$. Let $H_{v}^{k}=\left\{w \in H_{v}: h(w) \geq k\right\}$. Then $H_{v}^{k}$ are affine spaces as well and

$$
H_{v} \supseteq H_{v}^{1} \supseteq H_{v}^{2} \supseteq \ldots
$$


Hence for certain $k, H_{v}^{k}=H_{v}^{k+1}=\ldots$, therefore $H_{v}^{k} \subseteq V_{B_{1}(C)}^{\infty}$. This proves our lemma.

Lemma 8.2. $V_{C}=V_{C}^{\infty}$.

Proof. Let $v \in V_{C}^{\infty}$. Then by the previous lemma, there exist $v_{k} \in V_{B_{k}(C)}^{\infty}$ such that $\left.v_{k}\right|_{B_{k}(C)}=\left.v_{k+1}\right|_{B_{k}(C)}$. Hence the pointwise limit $l \in \prod_{\gamma \in \Gamma} K^{n}$ of $\left\{v_{k}\right\}_{k=1}^{\infty}$ exists. Then $\left.l\right|_{B_{k}(C)}=v_{k}$ and $\left.l\right|_{C}=\left.v\right|_{C}$. Hence by condition (4), $l \in V$ and $v \in V_{C}$.

Now we turn back to the proof of our proposition. Note that $V_{C}^{E} \supseteq V_{C}^{1} \supseteq$ $V_{C}^{2} \supseteq \ldots$ is a decreasing sequence of subspaces containing $V_{C}$. Hence for some $r$, $V_{C}^{r}=V_{C}^{r+1}=\ldots$ Thus $V_{C}^{r}=V_{C}^{\infty}$. Hence by our previous lemma $V_{C}^{r}=V_{C}$.

Proposition 8.2. Let $V^{E}$ be an extended configuration of the invariant subspace $V$, then

$$
\operatorname{dim}_{\Gamma}^{A}(V)=\operatorname{dim}_{\Gamma}^{A}\left(V^{E}\right)
$$

Proof. We need to show that for any $\delta>0$, there exists $Q>0$ such that if $q \geq Q$, then

$$
\frac{\operatorname{dim} V_{\mathcal{F}_{q}}^{E}}{\left|\mathcal{F}_{q}\right|} \leq(1+\delta) \operatorname{dim}_{\Gamma}^{A}(V)+\delta .
$$

Let $\epsilon$ be a real number; its exact value will be chosen at the end of the proof. By Proposition 6.1 we have integers $N \leq n_{1}<n_{2}<\cdots<n_{l}$ and $M>0$ such that $\left\{\mathcal{F}_{n_{1}}, \mathcal{F}_{n_{2}}, \ldots, \mathcal{F}_{n_{l}}\right\} \epsilon$-quasitile $\mathcal{G}_{m}$ for any $m \geq M$, and

$$
\frac{\operatorname{dim} V_{\mathcal{F}_{n_{i}}}}{\left|\mathcal{F}_{n_{i}}\right|} \leq \operatorname{dim}_{\Gamma}^{A}(V)+\epsilon, \quad \text { for any } 1 \leq i \leq k .
$$

By our previous lemma, there exists $r>0$ such that for any $1 \leq i \leq k$,

$$
V_{\mathcal{F}_{n_{i}}}^{r}=V_{\mathcal{F}_{n_{i}}} .
$$

Let $P_{1}, P_{2}, \ldots, P_{c_{s}}$ be those translated copies in the $\epsilon$-quasitiling of $\mathcal{F}_{s}$ such that $B_{r}\left(P_{i}\right) \subseteq \mathcal{F}_{s}$. Let $R_{i} \subseteq P_{i}$ denote the disjoint parts. Let $N_{s}$ be the cardinality of those points in $\mathcal{F}_{s}$ which are not covered by $\bigcup_{i=1}^{c_{s}} R_{i}$. Then $N_{s} \leq \epsilon^{2}\left|\mathcal{F}_{s}\right|+$ $\left|\partial_{r+D+1} \mathcal{F}_{s}\right|$, where $D=\max _{1 \leq i \leq k} \operatorname{diam}\left(\mathcal{F}_{n_{i}}\right)$. Then by the third condition in the definition of extended configurations,

$$
\operatorname{dim} V_{\mathcal{F}_{s}}^{E} \leq \sum_{i=1}^{c_{s}} \operatorname{dim} V_{R_{i}}+n\left(\epsilon^{2}\left|\mathcal{F}_{s}\right|+\left|\partial_{r+D+1} \mathcal{F}_{s}\right|\right)
$$

Hence,

$$
\operatorname{dim} V_{\mathcal{F}_{s}}^{E} \leq\left|\mathcal{F}_{s}\right|(1-\epsilon)^{-1}\left(\operatorname{dim}_{\Gamma}^{A}(V)+\epsilon\right)+n\left(\epsilon^{2}\left|\mathcal{F}_{s}\right|+\left|\partial_{r+D+1} \mathcal{F}_{s}\right|\right)
$$

Therefore, for large $s$,

$$
\frac{\operatorname{dim} V_{\mathcal{F}_{s}}^{E}}{\left|\mathcal{F}_{s}\right|} \leq(1-\epsilon)^{-1}\left(\operatorname{dim}_{\Gamma}^{A}(V)+\epsilon\right)+2 n \epsilon^{2} .
$$

Picking the right $\epsilon$ one can assert that (2) holds. 


\section{Additivity}

Proposition 9.1. Let $V \subseteq \prod_{\gamma \in \Gamma} K^{n}, W \subseteq \prod_{\gamma \in \Gamma} K^{m}$ be invariant subspaces. Let $T: V \rightarrow W$ be a continuous $\Gamma$-equivariant map. Then

$$
\operatorname{dim}_{\Gamma}^{A}(V)=\operatorname{dim}_{\Gamma}^{A}(\operatorname{Ker} T)+\operatorname{dim}_{\Gamma}^{A}(\overline{\operatorname{Ran} T}) .
$$

Proof. First note that both $\operatorname{Ker} T$ and $\overline{\operatorname{Ran} T}$ are invariant subspaces. By Proposition 3.1 it is given by matrix multiplication. Therefore $T$ has finite width, that is, there exists $L>0$ such that for any $\gamma \in \Gamma, T f(\gamma)=T g(\gamma)$, whenever $\left.f\right|_{B_{L}(\gamma)}=\left.g\right|_{B_{L}(\gamma)}$. For any finite subset $A \subseteq \Gamma$, let

$$
\begin{aligned}
N_{A}^{E}=\left\{v \in \prod_{\gamma \in \Gamma} K^{n}: \operatorname{supp} v \subseteq A \text { and there exists } w \in V_{B_{L}(A)}\right. \\
\text { such that } \left.\left.w\right|_{A}=\left.v\right|_{A} \text { and }\left.T v\right|_{A}=0\right\} .
\end{aligned}
$$

Then $N_{A}^{E}$ is an extended configuration of $\operatorname{Ker} T$. Also let

$$
\begin{aligned}
M_{A}^{E}=\left\{v \in \prod_{\gamma \in \Gamma} K^{n}: \operatorname{supp} v \subseteq A \text { and there exists } w \in V_{B_{L}(A)}\right. \\
\left.\quad \text { such that }\left.T w\right|_{A}=\left.v\right|_{A}\right\} .
\end{aligned}
$$

Then $M_{A}^{E}$ is an extended configuration of $\overline{\operatorname{RanT}}$. Let $R_{A}: V_{B_{L}(A)} \rightarrow \prod_{\gamma \in A} K^{m}$ be the restriction of $T$ onto $A$. Then $\operatorname{Ran}\left(R_{A}\right) \cong M_{A}^{E}$. Obviously, for any Følner-set $\mathcal{F}_{s}, \operatorname{dim} N_{\mathcal{F}_{s}}^{E} \leq \operatorname{dim} \operatorname{Ker} R_{\mathcal{F}_{s}}$. However, any element of $\operatorname{Ker} R_{\mathcal{F}_{s}}$ is determined by its restriction onto $\mathcal{F}_{s} \cup \partial_{r} \mathcal{F}_{s}$. Hence,

$$
\operatorname{dim} \operatorname{Ker} R_{\mathcal{F}_{s}} \leq \operatorname{dim} N_{\mathcal{F}_{s}}^{E}+n\left|\partial_{r} \mathcal{F}_{s}\right|
$$

Now,

$$
\operatorname{dim} \operatorname{Ker} R_{\mathcal{F}_{s}}+\operatorname{dim} \operatorname{Ran} R_{\mathcal{F}_{s}}=\operatorname{dim} V_{B_{L}\left(\mathcal{F}_{s}\right)} .
$$

Observe that

$$
\lim _{s \rightarrow \infty} \frac{\operatorname{dim} V_{B_{L}\left(\mathcal{F}_{s}\right)}}{\left|\mathcal{F}_{s}\right|}=\operatorname{dim}_{\Gamma}^{A}(V) .
$$

Also, by Proposition 8.2 and (3),

$$
\lim _{s \rightarrow \infty} \frac{\operatorname{dim} \operatorname{Ker} R_{\mathcal{F}_{s}}}{\left|\mathcal{F}_{s}\right|}=\operatorname{dim}_{\Gamma}^{A}(\operatorname{Ker} T)
$$

and

$$
\lim _{s \rightarrow \infty} \frac{\operatorname{dim} \operatorname{Ran} R_{\mathcal{F}_{s}}}{\left|\mathcal{F}_{s}\right|}=\operatorname{dim}_{\Gamma}^{A}(\overline{\operatorname{RanT}}) .
$$

Hence by (4) our proposition holds.

Proposition 9.2. Let $V \subseteq \prod_{\gamma \in \Gamma} K^{n}, W \subseteq \prod_{\gamma \in \Gamma} K^{m}$ and $Z \subseteq \prod_{\gamma \in \Gamma} K^{s}$ be invariant subspaces. Also let $T: V \rightarrow W$ and $S: W \rightarrow Z$ be continuous $\Gamma$ equivariant maps such that

$$
0 \rightarrow V \stackrel{T}{\rightarrow} W \stackrel{S}{\rightarrow} Z \rightarrow 0
$$

is weak-exact. Then,

$$
\operatorname{dim}_{\Gamma}^{A}(V)+\operatorname{dim}_{\Gamma}^{A}(Z)=\operatorname{dim}_{\Gamma}^{A}(W) .
$$

Proof. By our previous proposition, $\operatorname{dim}_{\Gamma}^{A}(\operatorname{Ker} S)=\operatorname{dim}_{\Gamma}^{A}(\overline{\operatorname{RanT}})=\operatorname{dim}_{\Gamma}^{A}(V)$ and $\operatorname{dim}_{\Gamma}^{A}(\operatorname{Ker} S)+\operatorname{dim}_{\Gamma}^{A}(Z)=\operatorname{dim}_{\Gamma}^{A}(W)$, which imply our proposition. 


\section{The END OF The PRoof of Theorem 1}

Let $M$ be a finitely generated module over $K \Gamma$. Define $r k(M)$ as $\operatorname{dim}_{\Gamma}^{A}\left(M^{*}\right)$. Then by Proposition $9.2 r k(M)=r k(N)$ if $M \cong N$, and $r k([K \Gamma])=1$. Now, let $0 \rightarrow M \rightarrow P \rightarrow N \rightarrow 0$ be an exact sequence of finitely generated modules. Then by Proposition 9.2 and Proposition $5.1 r k(P)=r k(N)+r k(M)$. This finishes the proof of our theorem.

\section{REFERENCES}

1. G. ElEK, Amenable groups, topological entropy and Betti numbers. (to appear in the Israel Journal of Mathematics)

2. W. LüCK, Dimension theory of arbitrary modules over finite von Neumann algebras and $L^{2}$ Betti numbers. II: Applications to Grothendieck groups, $L^{2}$-Euler characteristics and Burnside groups, J. Reine Angew. Math 496 (1998) 213-236. MR 99k:58177

3. D. S. ORnstein and B. Weiss, Entropy and isomorphism theorems for actions of amenable groups, J. Anal. Math 48 (1987) 1-141. MR 88j:28014

4. D. Ruelle, Thermodynamic formalism, Encyclopedia of Mathematics and Its Applications, Addison-Wesley 5 (1978) MR 80g:82017

Mathematical Institute of the Hungarian Academy of Sciences, P.O. Box 127, H-1364 BudAPEST, Hungary

E-mail address: elek@renyi.hu 